\title{
State Estimation and Motion Tracking for Spatially Diverse VLC Networks
}

\author{
M. Rahaim, G. B. Prince, T.D.C. Little \\ Department of Electrical and Computer Engineering \\ Boston University, Boston, MA 02215 \\ \{mrahaim,gbprince,tdcl\}@bu.edu
}

MCL Technical Report No. 09-14-2012

\begin{abstract}
Improvements in solid-state/LED lighting are driving increased capabilities of lighting systems offering potential for applications such as Visible Light Communications (VLC) and indoor localization using the lighting medium. In this paper, we motivate the adoption of localization for both the support of handover between arrays of VLC-equipped luminaires as well as for indoor positioning and beam steering. Our approach uses a state estimation model to achieve localization and motion tracking in spatially diverse VLC networks while considering user mobility and dynamic device orientation. Under these conditions, we show simulation results with location and velocity errors of $5 \mathrm{~cm}$ and $10 \mathrm{~cm} / \mathrm{s}$, respectively.
\end{abstract}

Keywords -- Visible Light Communication, Localization, Handover, State Estimation, Kalman Filter.

In Globecom Workshops - Optical Wireless Conference - Anaheim, CA, December 2012. This material is based upon work support by the National Science Foundation under Grant No. EEC-0812056. 


\section{Introduction}

Visible Light Communication (VLC) is experiencing growing interest due to improvements in solid state lighting, rapid growth in demand for wireless communication, and ubiquity of lighting systems in indoor environments. Beyond the ability to provide data rates above $100 \mathrm{Mb} / \mathrm{s}$, VLC offers the additional benefit of spatial diversity due to directionality of the optical medium. This diversity leads to spatial reuse and drastic bandwidth density $(\mathrm{Mb} / \mathrm{s} / \mathrm{m} 2)$ improvements in multiuser environments. Heterogeneous wireless systems have also been proposed where VLC supplements conventional RF communications in order to provide the bandwidth density benefits of VLC while mitigating issues related to channel blocking, intrusive uplink, and marketability [1], [2].

A major consideration for spatially diverse and heterogeneous systems is the optimization of handover as a user traverses the environment. Previous work has shown that appropriate handover decisions can decrease packet transfer delay and improve overall QoS by reducing the number of unnecessary channel transfers [3]. This can include Horizontal Handover (HHO) where traffic is transferred between VLC channels (e.g., between luminaires) or Vertical Handover (VHO) where traffic is transferred to an alternate medium (e.g., RF). State estimation techniques provide a method to track the motion of a user in the physical space and to predict their path - facilitating handover through predictive methods which minimize the number of channel transfers when compared to techniques that monitor signal strength alone [4]. Predicting device location has also been discussed as a means of steering high data rate VLC channels for improved performance [5].

Beyond the potential for assisted communication, localization, or positioning, has been a subject of growing interest for years as mobile computing is becoming the norm in society. Many of the functions that mobile services provide rely heavily on mobile device position - primarly using GPS for outdoor scenarios. The research field has been flooded with many attempts at balancing performance, cost, and complexity of indoor positioning systems. There have been two main high level approaches set forth to solve the indoor localization problem: (1) exploiting existing infrastructure and developing algorithms to perform the best possible given the available infrastructure and its constraints (e.g., WiFi signal strength, fluorescent lighting) or (2) designing specialized solutions that address the fundamental issues that are core to the localization problem using the best technologies to solve the problem. Localization with VLC provides a compromise.

In this paper, we propose and analyze a state estimation model for tracking device location and motion in an environment with fixed position VLC transmitters and mobile users. Section II provides the necessary background description of the VLC channel and its constraints while surveying benefits of indoor localization and Kalman Filter recursive estimation. Section III describes the observed system model that we use to analyze the benefits of recursive estimation techniques for motion tracking in VLC enabled environments. Section IV provides the results in terms of accuracy and time to convergence, while Section V concludes the investigation.

\section{Background}

\subsection{Visible Light Communication Channel}

Optical communication has been studied across the spectrum and in both constrained and free-space for many years. Improvements in solid state lighting have made the visible spectrum fertile ground for extending this research field in the form of VLC. Due to signal directionality, path loss in an optical channel such as VLC is modeled differently than that of an RF channel. Angles of transmission and 
acceptance effect received signal power; therefore additional parameters are observed. The received signal at receiver $\mathrm{j}$ from transmitter i can be modeled as

$$
P_{r, i j}=\frac{P_{t, i} T\left(\phi_{i j}\right) A_{r} g\left(\theta_{i j}\right)}{D_{i j}^{2}}
$$

where $P_{t}$ is the transmitted optical power, $A_{r}$ is the area of the receiver photodiode, $D_{i j}$ is the distance between transmitter and receiver, and $\phi_{i j}$ and $\theta_{i j}$ represent angles of emission and acceptance, respectively. The intensity pattern of a typical LED is modeled as a Lambertian pattern with order n, given by

$$
T(\phi)=\frac{n+1}{2 \pi} \cos ^{n}(\phi)
$$

and the optical gain function for a bare photodiode can generally be observed as $g(\theta)=\cos (\theta)[6]$. Overlapping channels can be distinguished through channel separation techniques such as time division, frequency division, code division, or wavelength division [7].

\subsection{Indoor Localization}

Indoor localization has been explored in many contexts, but most often finds application in piggybacking existing communication infrastructures (e.g., WiFi, Bluetooth) due to the fact that customized solutions (e.g., UWB, Ultrasound, and IR) are too expensive and require hardware devoted solely to localization in addition to existing communication infrastructure.Moreover, localization in the conventional sense requires transceivers capable of processing channel measurements (e.g., angle, signal strength, time of flight) and relate them to positional estimates through localization algorithms (e.g., multilateration, triangulation). With the transition from electric to electronic lighting and the ubiquity of light in indoor spaces, visible light may prove to be the next communication infrastructure to exploit for localization services.

The benefits of visible light as a localization medium are its directionality, short range, and impulse response, while its issues to overcome are installation accuracy, network layer identification, coexistence of WiFi and VLC, and efficient multiple access schemes. Visible light is one of the most viable solutions to indoor positioning due to its directionality, short impulse response, and the distribution and availability of anchor luminaries to meet the illumination needs of indoor spaces. One approach exploits the ubiquity and directionality of light in order to localize the mobile user [8]. In this work, we observe measurements of the optical channel as part of a recursive state estimation model with the goal of estimating dynamic position and velocity of a user device.

\subsection{Kalman Filtering}

Generally, discrete-time linear state space models are employed to describe the behavior of dynamical systems

$$
\begin{gathered}
\boldsymbol{x}[t+1]=\boldsymbol{A} x[t]+\boldsymbol{B} u[t]+\boldsymbol{G} w[t] \\
y[t]=\boldsymbol{C} x[t]+\boldsymbol{D} u[t]+\boldsymbol{H} v[t]
\end{gathered}
$$

The discrete-time Kalman Filter (KF) is a recursive MMSE estimator, and for the purpose of this investigation, its operation can be summarized into a few high-level steps: initialization, prediction, measurement, and update. The filter requires an initial state and covariance estimate to begin operation. 
It uses this initial estimate, which can be any time instant $t$ (not necessarily zero) ( $\boldsymbol{x}_{t \mid t}$ and $\boldsymbol{\Sigma}_{t \mid t}$ ) to predict the state, $\boldsymbol{x}_{t+1 \mid t}$, and covariance, $\boldsymbol{\Sigma}_{t+1 \mid t}$, at the next time step. The filter then receives measured data, $\boldsymbol{y}_{t+1}$, from the environment and compares it to the predicted measurement, $\boldsymbol{y}_{t+\mid t}$; this difference is known as the innovations of the filter, $\boldsymbol{v}_{t+1}$. The innovations provide the filter sufficient information to adjust its "Kalman Gain," $\boldsymbol{K}_{t+1}$, to correct its inaccuracies between its predicted and measured data during its update state. The filter is recursive in the sense that it will use this last update as its reference for making a prediction in the subsequent time step and is summarized mathematically as:

$$
\begin{gathered}
\boldsymbol{x}_{t+1 \mid t}=\boldsymbol{A} \boldsymbol{x}_{t \mid t}+\boldsymbol{B} u[t]+\boldsymbol{G} E\{w[t]\} \\
\boldsymbol{\Sigma}_{t+1 \mid t}=\boldsymbol{A} \boldsymbol{\Sigma}_{t \mid t} \boldsymbol{A}^{T}+\boldsymbol{G} \boldsymbol{Q} \boldsymbol{G}^{T} \\
\boldsymbol{y}_{t+1 \mid t}=\boldsymbol{C} \boldsymbol{x}_{t \mid t}+\boldsymbol{D} u[t]+\boldsymbol{H} E\{v[t]\} \\
\boldsymbol{x}_{t+1 \mid t+1}=\boldsymbol{x}_{t+1 \mid t}+\boldsymbol{K}_{t+1} \boldsymbol{v}_{t+1} \\
\boldsymbol{\Sigma}_{t+1 \mid t+1}=\left[\boldsymbol{I}-\boldsymbol{C} \boldsymbol{K}_{t+1}\right] \boldsymbol{\Sigma}_{t+1 \mid t} \\
\boldsymbol{v}_{t+1}=\boldsymbol{y}_{t+1}-\boldsymbol{y}_{t+1 \mid t} \\
\boldsymbol{K}_{t+1}=\boldsymbol{\Sigma}_{t+1 \mid t} \boldsymbol{C}^{T}\left[\boldsymbol{C} \boldsymbol{\Sigma}_{t+1 \mid t} \boldsymbol{C}^{T}+R\right]^{-1}
\end{gathered}
$$

The basic KF operates on the assumption that all system dynamics and measurement models are linear; however many circumstances arise in which these relations are non-linear. Linearization of the nonlinear model creates the discrete-time linear form known as the Extended Kalman Filter (EKF) [9]. Depending on the degree of non-linearity, the EKF is difficult to implement, difficult to tune, and only reliable for systems that are almost linear on the time scale of the updates due to the inherent linearization in the EKF.

An alternative approach is to use an Unscented Transformation (UT) [10]. The principle behind the UT is that it is easier to approximate a probability distribution than it is to approximate an arbitrary nonlinear transformation. The UT requires the selection of a set of $\sigma$-points which deterministically (contrary to random particle filters) mirror the mean and covariance of the states of the system. Furthermore, weights are assigned to the $\sigma$-points to provide an unbiased estimator. The mean and covariance of the $\sigma$-points can be used in the KF to produce the MMSE of the state. The UT propagates system state and covariance information through nonlinear transformations rather than analytically linearizing the transformation. It is more accurate, easier to implement, and uses the same order of calculations as linearization.

\section{System Description}

In this section, we describe the environment under consideration, the observed KF model, and three scenarios simulated in order to assess the accuracy of the model. Although we discuss the implementation for a defined environment, the concept may be extended to generic environments with known transmitter locations and orientations.

\subsection{System Model}

As a base case for the model, we observe an empty room with dimensions $6 \mathrm{~m} \mathrm{X} \mathrm{6m} \mathrm{X} \mathrm{4m} \mathrm{and} \mathrm{fixed}$ position transmitters located in the grid layout or cellular layouts shown in Figure 1. The grid layout is a conventional layout for recessed lighting fixtures (luminaires) and the cellular layout follows the 

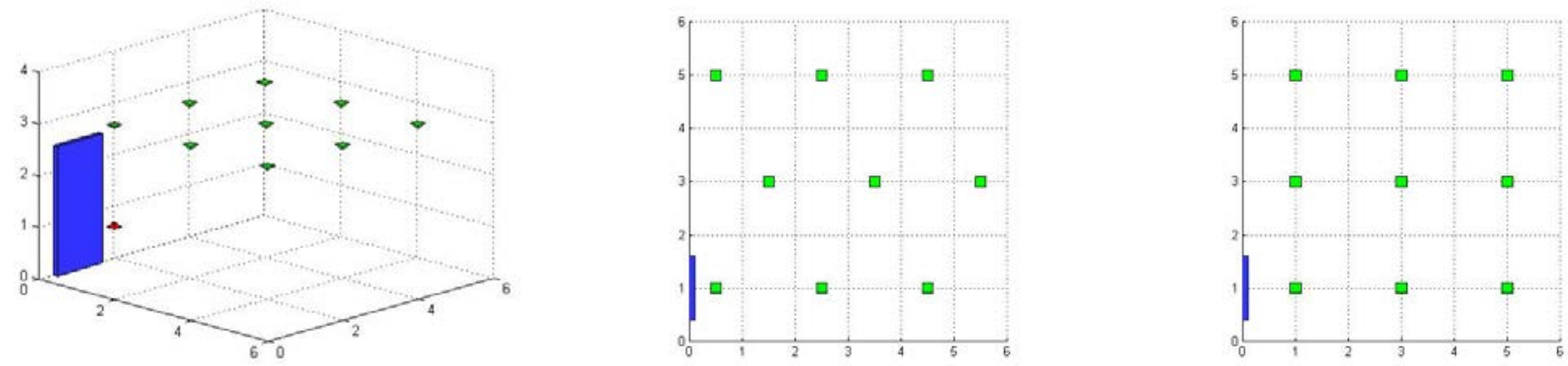

Figure 1: Reference room with overhead view of grid and cellular layouts

hexagonal structure of classic cellular networks. We assume that a user first determines the transmitter with maximum signal strength and that their initial location in the environment is normally distributed with expected value below the transmitter. It is assumed that the user is moving through the environment with zero mean random acceleration in the $\mathrm{x}$ and $\mathrm{y}$ planes, following conventional state models for translational motion [11].

The KF model observed in this work considers a linear state model, $\boldsymbol{x}$, with transition matrix, $\boldsymbol{A}$, and a nonlinear measurement, $\boldsymbol{y}$. Process noise, $w$, and measurement noise, $\boldsymbol{v}$, are assumed to be independent, zero-mean, Gaussian white noise processes with covariance matrices $\boldsymbol{Q}$ and $\boldsymbol{R}$, respectively.

$$
\begin{gathered}
\boldsymbol{x}[t+1]=\boldsymbol{A} x[t]+w[t] \\
\boldsymbol{y}[t]=h[\boldsymbol{x}[t], t]+v[t]
\end{gathered}
$$

\subsection{Scenario I}

In the first scenario, we observe a receiver directed perpendicular to the floor such that $\phi_{i j}=\theta_{i j}$. The state represents the position and velocity in the $\mathrm{x}$ and $\mathrm{y}$ planes and location in the $\mathrm{z}$ plane is assumed to be held constant. The measurement vector consists of the set of received signal powers from each of the 9 transmitters in the room, including measurement noise. The signal from each transmitter is assumed to be distinguishable.

$$
\begin{gathered}
\boldsymbol{x}=\left[x, V_{x}, y, V_{y}\right]^{\prime} \\
\boldsymbol{y}=\left[S I G_{1 j}, S I G_{2 j}, \ldots, S I G_{9 j}\right]^{\prime}
\end{gathered}
$$

Scenario I observes the state transition and covariance matrices described in [11]. We then modify these matrices to account for additional parameters in scenarios II and III.

$$
\boldsymbol{A}_{I}=\left[\begin{array}{cccc}
1 & d t & 0 & 0 \\
0 & 1 & 0 & 0 \\
0 & 0 & 1 & d t \\
0 & 0 & 0 & 1
\end{array}\right] \quad \boldsymbol{Q}_{I}=q \cdot\left[\begin{array}{cccc}
\frac{d t^{3}}{3} & \frac{d t^{2}}{2} & 0 & 0 \\
\frac{d t^{2}}{2} & d t & 0 & 0 \\
0 & 0 & \frac{d t^{3}}{3} & \frac{d t^{2}}{2} \\
0 & 0 & \frac{d t^{2}}{2} & d t
\end{array}\right] \quad \boldsymbol{R}_{I}=r_{s i g} \cdot I_{9 x 9}
$$


As indicated by Equation 1, the received power from transmitter $i$ is dependent on $P_{t}, A_{r}, \phi_{i j}, \theta_{i j}$, and $D_{i j}$. The transmit power and receiver effective area are held constant, while $\phi_{i j}$, $\theta_{i j}$, and $D_{i j}$ are dependent on the receiver location in the $\mathrm{x}, \mathrm{y}$, and $\mathrm{z}$ planes.

$$
\begin{gathered}
D_{i j}^{2}=\left(X_{i}-x\right)^{2}+\left(Y_{i}-y\right)^{2}+\left(Z_{i}-z\right)^{2} \\
\phi_{i j}=\theta_{i j}=\arctan \left(\frac{\sqrt{\left(X_{i}-x\right)^{2}+\left(Y_{i}-y\right)^{2}}}{Z_{i}-z}\right)
\end{gathered}
$$

\subsection{Scenario II}

In the second scenario, we observe similar measurements while adding the additional complexity of device rotation to the state model. The state transition and process noise covariance matrices are also updated in order to account for the changes in the state model.

$$
\begin{gathered}
\boldsymbol{x}=\left[x, V_{x}, y, V_{y}, \theta_{e l}, \theta_{a z}\right]^{\prime} \\
\boldsymbol{A}_{I I}=\left[\begin{array}{ccc}
A_{I} & \overline{0} \\
\overline{0} & {\left[\begin{array}{ll}
1 & 0 \\
0 & 1
\end{array}\right]}
\end{array}\right] \quad \boldsymbol{Q}_{I I}=\left[\begin{array}{ccc}
Q_{I} & \overline{0} \\
\overline{0} & {\left[\begin{array}{cc}
\left(q_{\theta}\right) d t & 0 \\
0 & \left(q_{\theta}\right) d t
\end{array}\right]}
\end{array}\right]
\end{gathered}
$$

In this scenario, the assumption that $\phi_{i j}=\theta_{i j}$ no longer holds. The acceptance angle, $\theta_{i j}$, is now dependent on the rotation of the receiver. In order to determine $\theta_{i j}$, we observe the unit vector pointing away from from the center of the receiver, $V_{r x}$, and the unit vector pointing from the receiver to the $i$ th transmitter, $V_{t x, i}$. Given the two unit vectors, the acceptance angle can be calculated as shown below.

$$
\begin{gathered}
V_{r x}=\left\{\cos \left(\theta_{e l}\right) \cdot \sin \left(\theta_{a z}\right), \sin \left(\theta_{e l}\right) \cdot \sin \left(\theta_{a z}\right), \cos \left(\theta_{a z}\right)\right\} \\
V_{t x, i}=\left\{\left(X_{i}-x\right),\left(Y_{i}-y\right),\left(Z_{i}-z\right)\right\} \\
\cos \left(\theta_{i j}\right)=\frac{V_{r x} \cdot V_{t x, i}}{\left|V_{r x}\right|\left|V_{t x, i}\right|}
\end{gathered}
$$

\subsection{Scenario III}

In the final scenario, we aim to improve accuracy by observing device rotation in the measurement vector, $\boldsymbol{Y}$. This change to the measurement vector also has associated updates to the measurement noise covariance matrix.

$$
\begin{gathered}
\boldsymbol{y}=\left[S I G_{1 j}, S I G_{2 j}, \ldots, S I G_{9 j}, \theta_{e l}, \theta_{a z}\right]^{\prime} \\
\boldsymbol{R}_{I I I}=\left[\begin{array}{cc}
\boldsymbol{R}_{I} & \overline{0} \\
\overline{0} & r_{\theta} \cdot I_{2 x 2}
\end{array}\right]
\end{gathered}
$$

In this scenario, we aim to show that additional sensors, such as an accelerometer and gyroscope, can improve the performance of the algorithm in a realistic setting. 
Table 1

\section{Results}

The following section shows results of the three scenarios described above. We run a Monte Carlo simulation with 200 trials. A random path through the environment is observed for each trial and the error between the actual state and filter estimation is averaged across trials. Table I provides a list of parameter values observed in simulation. We will first present a set of Cramer Rao bounds, and then provide simulation results showing average error for varying parameters.

\subsection{Cramer Rao Bounds}

The first observation in Figure 2 shows the CRB across multiple sampling rates. The results show that the bounds on the estimates improve as the sampling rate increases. Figure 3 shows a similar comparison as the transmitter Lambertian order is increased from $n=1$ to $n=4$,

\begin{tabular}{|c|c|c|}
\hline Parameter & Phase I & Phase II / III \\
\hline \multicolumn{3}{|c|}{ System Parameters } \\
\hline$P_{t}(\mathrm{~W})$ & 5 & 5 \\
\hline$A_{r}\left(\mathrm{~mm}^{2}\right)$ & 300 & 300 \\
\hline$d t(\mathrm{~ms})$ & $0.2,0.5,1$ & 0.2 \\
\hline$n$ & $1,2,3,4$ & 1 \\
\hline \multicolumn{3}{|c|}{ Initial Expectation } \\
\hline $\mathrm{E}[x, y](\mathrm{m})$ & {$[0.99,0.99]$} & {$[0.99,0.99]$} \\
\hline $\mathrm{E}\left[V_{x}, V_{y}\right](\mathrm{m} / \mathrm{s})$ & {$[0.8,0.8]$} & {$[0.8,0.8]$} \\
\hline $\mathrm{E}\left[\theta_{e l}, \theta_{a z}\right]\left({ }^{\circ}\right)$ & - & {$[0,0]$} \\
\hline$\Sigma[x, y](\mathrm{m})$ & {$[0.5,0.5]$} & {$[0.5,0.5]$} \\
\hline$\Sigma\left[V_{x}, V_{y}\right](\mathrm{m} / \mathrm{s})$ & {$[0.2,0.2]$} & {$[0.2,0.2]$} \\
\hline$\Sigma\left[\theta_{e l}, \theta_{a z}\right]\left({ }^{\circ}\right)$ & - & {$\left[\frac{\pi}{180}, \frac{\pi}{4}\right]$} \\
\hline \multicolumn{3}{|c|}{ Noise Parameters } \\
\hline$r_{s i g}$ & $3.5 \cdot 10^{-8}$ & $3.5 \cdot 10^{-8}$ \\
\hline$r_{\theta}$ & - & $\frac{\pi}{360}$ \\
\hline$q$ & $10^{-2}$ & $10^{-2}$ \\
\hline$q_{\theta}$ & - & $\frac{\pi}{360}$ \\
\hline
\end{tabular}
corresponding to transmitter FOV ranging from $60^{\circ}$ to $35^{\circ}$. The bounds improve as the emission moves more towards a spot light.

The error bound on position converges quickly as it is directly related to the measurement; however error bound on velocity converges slower due to its relation to change in position. With a sampling period of $0.2 \mathrm{~ms}$ and Lambertian order $n=4$, the error bound on position converges to $1 \mathrm{~cm}$ after $100 \mathrm{~ms}$ and the error bound on velocity converges to $2 \mathrm{~cm} / \mathrm{s}$ after approximately $750 \mathrm{~ms}$.

\subsection{Kalman Filter Results}

For comparison of filters, Figures 4 and 5 show the simulation accuracies of the EKF and UT across the range of transmitter Lambertian orders for the grid layout with a sampling period of $0.2 \mathrm{~ms}$. We also show the CRB at $n=4$ for comparison. The high initial performance of the EKF is due to the initial distribution of the receiver location being centered around the region directly below a transmitter in the grid layout. The overhead transmitter provides a dominant signal - increasing the signal to noise ratio and providing very good accuracy. EKF performance degrades as receivers move away from the center

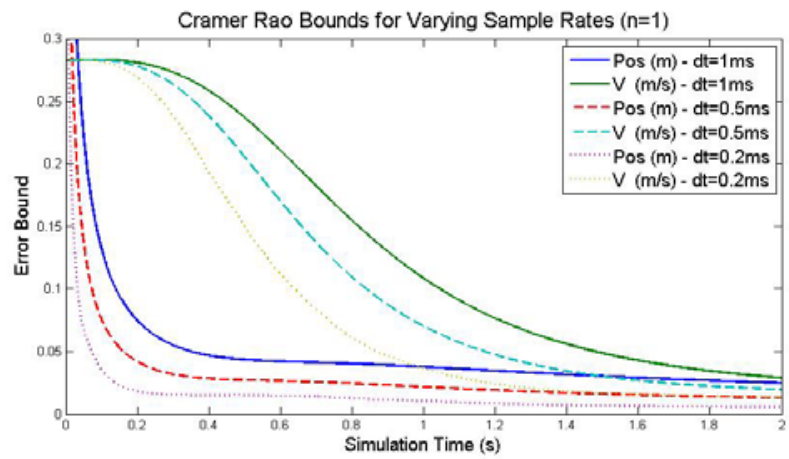

Figure 3: Cramer Rao Bounds for multiple sampling rates

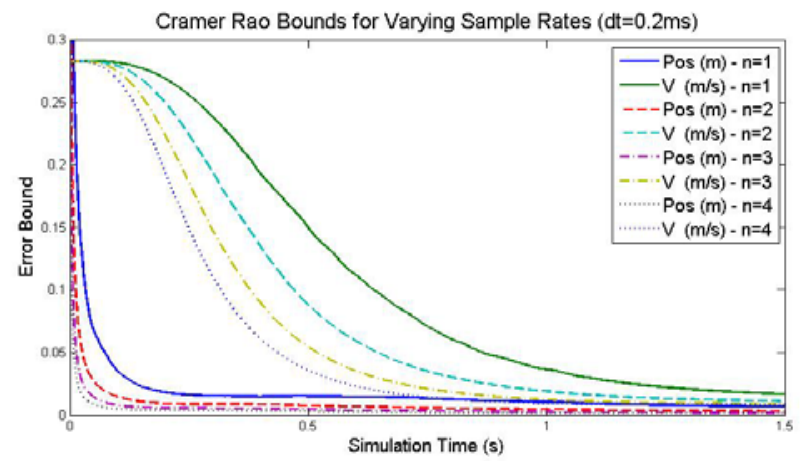

Figure 3: Cramer Rao Bounds for multiple transmitter orders 


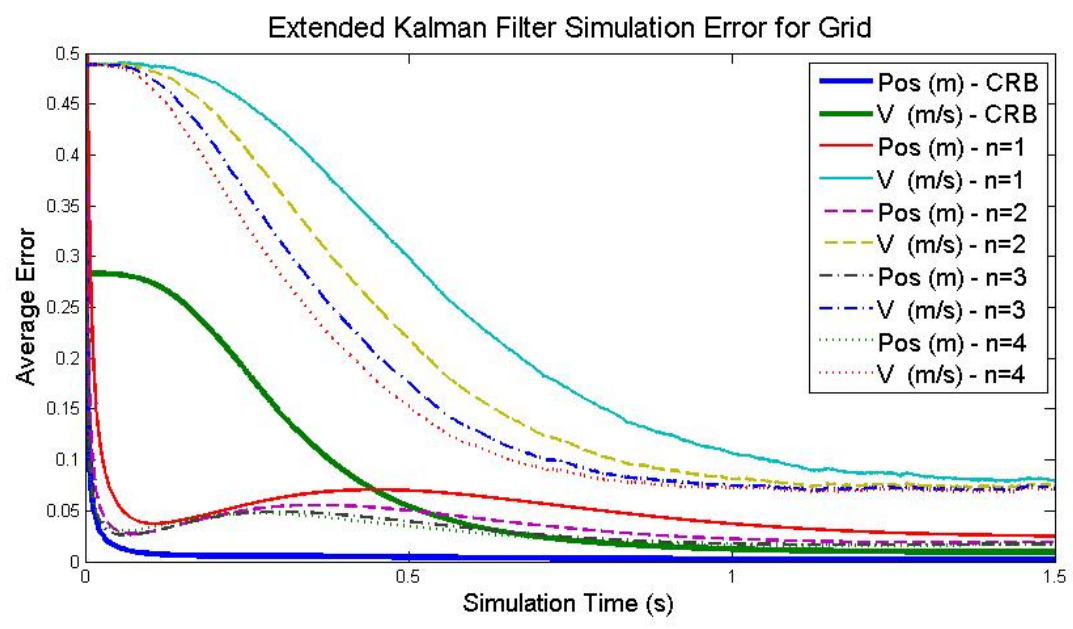

Figure 4: Simulation results for the Extended Kalman Filter

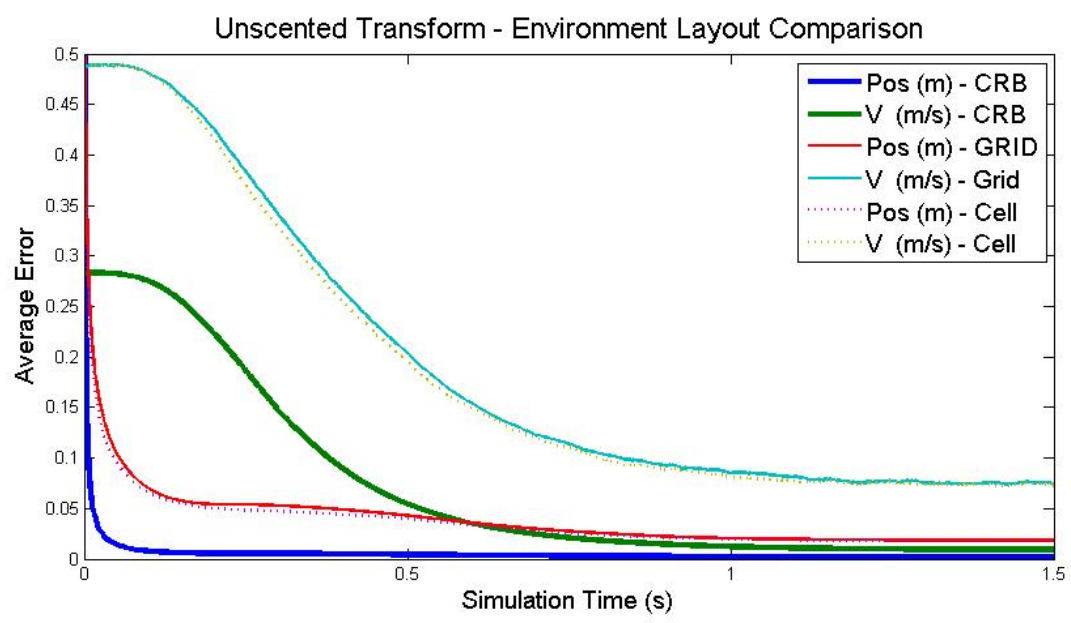

Figure 5: Simulation results for the Unscented Filter

of a transmitter and converges with the results from the UT as receivers move through the regions where the transmitter emission patterns provide very little overlap. In general, the EKF performed better when starting below a transmitter; however, we observed that the UT provides smoother results when the receiver is between transmitters - as with the case of the cellular layout. While the results shown here indicate narrow FOV transmitters provide better results, we have observed that there is an optimal setting between wide FOV transmitters and narrow FOV spot lights. As receivers move away from a transmitter, wide FOV implementations provide overlapping signals - leading to smooth transitions between dominant signals. Alternatively, very narrow FOV implementations have many low signal regions as the user traverses the room. In addition, lighting constraints limit the FOV of the transmitter as the typical environment requires near-uniform lighting throughout a room.

We also provide a performance comparison of the grid and cellular layouts for the UT in Figure 6. Although the cellular layout only shows slightly improved performance, it is important to note that the initial distribution of the user in the cellular environment is no longer centered under a transmitter since we compare against the same set of simulated motion paths. As previously discussed, the dominant signal at a receiver directly aligned with a transmitter impacts convergence time. 


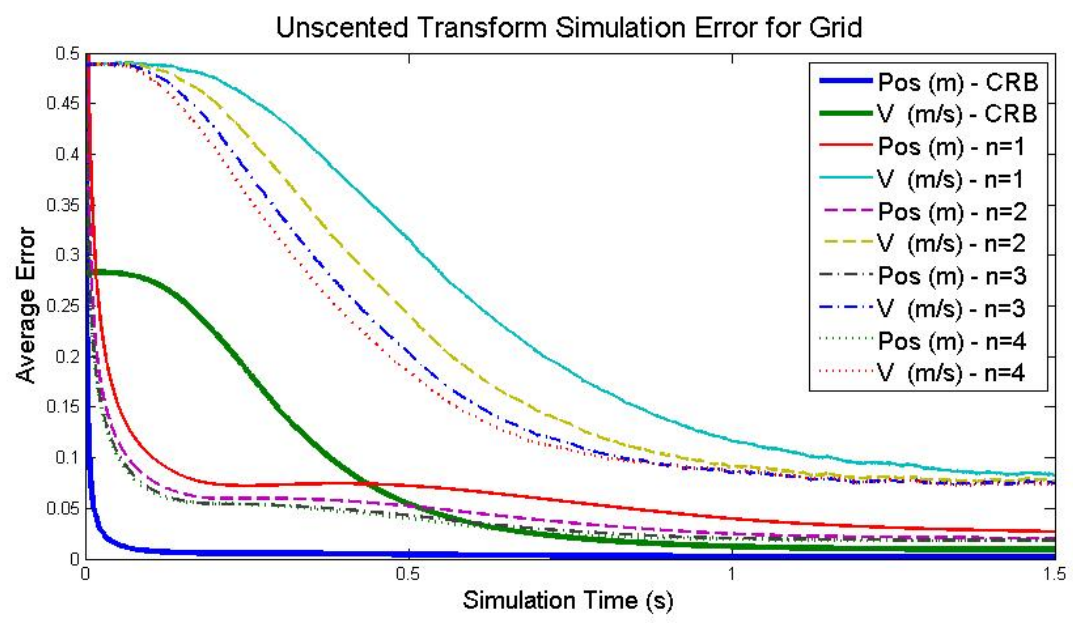

Figure 7: Simulation results comparing grid and cellular layouts

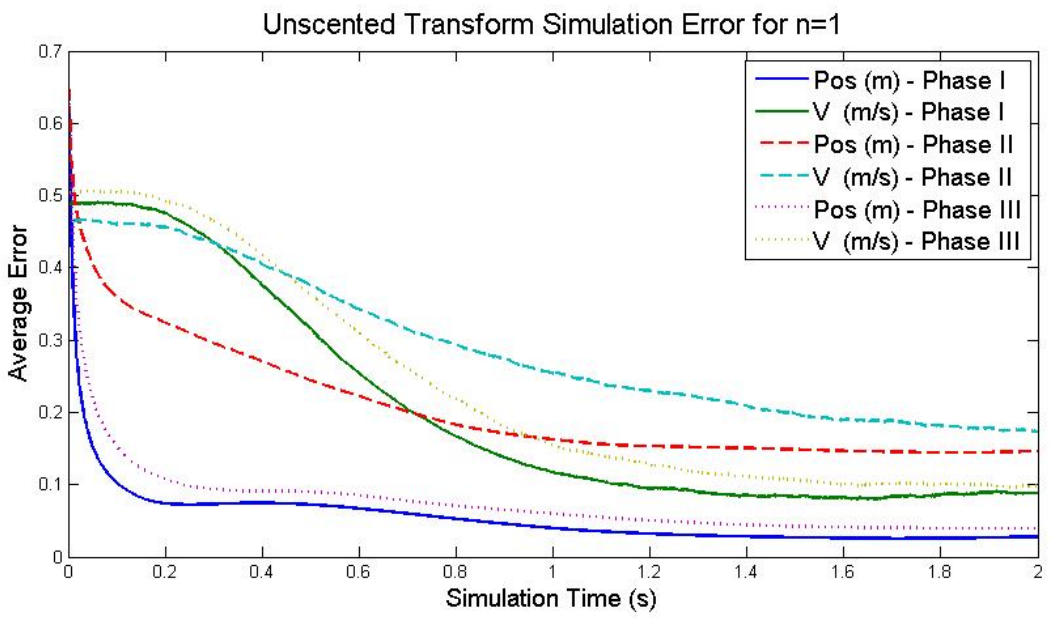

Figure 6: Simulation results comparing scenarios I, II, and III

Up until this point, the results shown have been under the base case assumptions from Scenario I. Figure 7 compares these idealistic results to the more realistic scenarios accounting for device rotation. Both position and velocity estimations are drastically degraded in Scenario II due to the additional degrees of freedom associated with user rotation; however, Scenario III shows that noisy measurements of this rotation can improve the position and velocity estimations such that the error is near that of the idealized base case.

\section{Conclusions}

In summary, we have provided a novel state estimation model leveraging the lighting infrastructure and capabilities of solid state lighting in order to approximate user location and motion under realistic conditions. We have also presented simulated results showing position and velocity estimations in an empty room with average error of approximately $5 \mathrm{~cm}$ and $10 \mathrm{~cm} / \mathrm{s}$, respectively.

We have accounted for realistic conditions including mobile users and device rotation; however we recognize that additional complexities in an environment occur due to dynamic signal conditions from obstructions and signal reflections. If the device is controlled by a user, it is likely that the user will 
occlude the signal from one direction; however we claim that this occlusion may be used to provide additional information to the system, assuming the motion path is known to be in the direction of the unobstructed signals. We have also discussed how a dominant signal has a major impact on performance - indicating that valid results may still be obtainable if some channels are blocked.

The discussion presented in this report provides a method for tracking device location and motion. Moving forward, such results are applicable in many use cases including indoor positioning systems and asset tracking as well as assisted handover methods and beam steering for indoor VLC networks.

\section{References}

[1] D. O'Brien. Cooperation in Optical Wireless Communications. Cognitive Wireless Networks, pages 623-634, 2007.

[2] M. Rahaim, A.M. Vegni, and T.D.C. Little. A Hybrid Radio Frequency and Broadcast Visible Light Communication System. IEEE Workshop on Optical Wireless Communications, pages 818822, 2011.

[3] J. Hou and D O’Brien. Vertical Handover-Decision-Making Algorithm Using Fuzzy Logic for the Integrated Radio-and-OW System. Wireless Communications, IEEE Transactions on, 5(1):176 185, Jan. 2006.

[4] G.P. Pollini. Trends in Handover Design. IEEE Communications Magazine, 34(3):82-90, 1996.

[5] G. Cossu, M. Presi, R. Corsini, P. Choudhury, A.M. Khalid, and E. Ciaramella. A Visible Light localization Aided Optical Wireless System. In GLOBECOM Workshops (GC Wkshps), 2011 IEEE, pages $802-807$, dec. 2011.

[6] J.B. Carruthers, S.M. Carroll, and P. Kannan. Propagation Modelling for Indoor Optical Wireless Communications Using Fast Multi-Receiver Channel Estimation. Optoelectronics, IEE Proceedings -, 150(5):473 - 481, oct. 2003.

[7] H. Elgala, R. Mesleh, H. Haas, and B. Pricope. OFDM Visible Light Wireless Communication Based on White LEDs. In Vehicular Technology Conference, 2007. VTC2007-Spring. IEEE 65th, pages 2185 - 2189, april 2007.

[8] G. B. Prince and T.D.C. Little. A Two Phase Hybrid RSS/AoA Algorithm for Indoor Device Localization using Visible Light. Globecom, 2012.

[9] R.K. Mehra. A Comparison of Several Nonlinear Filters for Re-entry Vehicle Tracking. In Adaptive Processes (9th) Decision and Control, 1970. 1970 IEEE Symposium on, volume 9, page 194, dec. 1970.

[10] S.J. Julier and J.K. Uhlmann. Unscented Filtering and Nonlinear Estimation. Proceedings of the IEEE, 92(3):401 - 422, mar 2004.

[11] Markus Kohler. Using the Kalman Filter to Track Human Interactive Motion - Modelling and Initialization of the Kalman Filter for Translational Motion. Technical report, 1997. 